Bull. Korean Math. Soc. 51 (2014), No. 1, pp. 139-156

http://dx.doi.org/10.4134/BKMS.2014.51.1.139

\title{
ERROR ESTIMATES OF RT1 MIXED METHODS FOR DISTRIBUTED OPTIMAL CONTROL PROBLEMS
}

\author{
TiAnliang Hou
}

\begin{abstract}
In this paper, we investigate the error estimates of a quadratic elliptic control problem with pointwise control constraints. The state and the co-state variables are approximated by the order $k=1$ Raviart-Thomas mixed finite element and the control variable is discretized by piecewise linear but discontinuous functions. Approximations of order $h^{\frac{3}{2}}$ in the $L^{2}$-norm and order $h$ in the $L^{\infty}$-norm for the control variable are proved.
\end{abstract}

\section{Introduction}

In the recent years, the finite element approximation plays an important role in the numerical treatment of optimal control problems. This approach has been extensively studied in $[9,16]$. In particular, a priori error estimates of finite element approximations for optimal control problems governed by linear elliptic equations were established in, for example, $[1,12,13]$, superconvergence and a posteriori error estimates have been discussed in e.g., [2, 6, 14, 17, 19, 20]. Note that all the above works aimed at standard finite element method.

For the mixed finite element approximations of optimal control problems, Chen et al. have done some works on priori error estimates and superconvergence properties of mixed finite elements for optimal control problems, see $[4,5,7,8]$. Recently, in [22], Xing and Chen have analyzed the $L^{\infty}$-error estimates for general convex optimal control problems with the lowest order Raviart-Thomas mixed finite element methods, while the $L^{\infty}$-error estimates for quadratic optimal control problems governed by semilinear elliptic equations was investigated in [18].

In this paper, the state and the co-state variables are approximated by order $k=1$ Raviart-Thomas mixed finite element spaces and the control is approximated by piecewise linear but discontinuous functions. We derive the $L^{2}$ - and

Received December 24, 2012.

2010 Mathematics Subject Classification. 49J20, 65N30.

Key words and phrases. elliptic equations, distributed optimal control problems, $L^{\infty}$ error estimates, RT1 mixed finite element methods. 
$L^{\infty}$-error estimates for the control variable. We consider the following linearquadratic optimal control problems for the state variables $\boldsymbol{p}, y$, and the control $u$ involving pointwise control constraints:

$$
\min _{u \in U_{a d}}\left\{\frac{1}{2}\left\|\boldsymbol{p}-\boldsymbol{p}_{d}\right\|^{2}+\frac{1}{2}\left\|y-y_{d}\right\|^{2}+\frac{\nu}{2}\|u\|^{2}\right\}
$$

subject to the state equation

$$
-\operatorname{div}(A(x) \operatorname{grad} y)+a_{0} y=f+u, \quad x \in \Omega,
$$

which can be written in the form of the first order system

$$
\operatorname{div} \boldsymbol{p}+a_{0} y=f+u, \quad \boldsymbol{p}=-A(x) \operatorname{grad} y, \quad x \in \Omega,
$$

and the boundary condition

$$
y=0, \quad x \in \partial \Omega,
$$

where $\Omega$ is a bounded domain in $\mathbb{R}^{2}$. $U_{a d}$ denotes the admissible set of the control variable, defined by

$$
U_{a d}=\left\{u \in L^{2}(\Omega): a \leq u \leq b, \text { a.e. in } \Omega\right\},
$$

where $a$ and $b$ are two real numbers that fulfill $a<b$. Moreover, we assume that $0 \leq a_{0} \in W^{1, \infty}(\Omega), f, y_{d}$ and $\boldsymbol{p}_{d}$ are given functions. $\nu$ is a fixed positive number. The coefficient $A(x)=\left(a_{i j}(x)\right)$ is a symmetric matrix function with $a_{i j}(x) \in W^{1, \infty}(\Omega)$, which satisfies the ellipticity condition

$$
c_{*}|\xi|^{2} \leq \sum_{i, j=1}^{2} a_{i j}(x) \xi_{i} \xi_{j}, \quad \forall(\xi, x) \in \mathbb{R}^{2} \times \bar{\Omega}, \quad c_{*}>0 .
$$

The plan of this paper is as follows. In Section 2, we construct the mixed finite element approximation scheme for the optimal control problem (1.1)(1.4) and give its equivalent optimality conditions. The main results of this paper are stated in Section 3, we first derive the $L^{2}$-error estimates for the optimal control problems, then we consider the $L^{\infty}$-error estimates for the control variable. In Section 4, we present a numerical example to demonstrate our theoretical results. In the last section, we briefly summarize the results obtained and some possible future extensions.

In this paper, we adopt the standard notation $W^{m, p}(\Omega)$ for Sobolev spaces on $\Omega$ with a norm $\|\cdot\|_{m, p}$ given by $\|v\|_{m, p}^{p}=\sum_{|\alpha| \leq m}\left\|D^{\alpha} v\right\|_{L^{p}(\Omega)}^{p}$, a seminorm $|\cdot|_{m, p}$ given by $|v|_{m, p}^{p}=\sum_{|\alpha|=m}\left\|D^{\alpha} v\right\|_{L^{p}(\Omega)}^{p}$. We set $W_{0}^{m, p}(\Omega)=\{v \in$ $\left.W^{m, p}(\Omega):\left.v\right|_{\partial \Omega}=0\right\}$. For $p=2$, we denote $H^{m}(\Omega)=W^{m, 2}(\Omega), H_{0}^{m}(\Omega)=$ $W_{0}^{m, 2}(\Omega)$, and $\|\cdot\|_{m}=\|\cdot\|_{m, 2},\|\cdot\|=\|\cdot\|_{0,2}$. In addition $C$ or $c$ denotes a general positive constant independent of $h$, where $h$ is the spatial mesh-size for the control and state discretization. 


\section{Mixed methods for optimal control problems}

In this section we shall construct mixed finite element approximation scheme of the control problem (1.1)-(1.4). For sake of simplicity, we assume that the domain $\Omega$ is a convex polygon. Now, we introduce the co-state elliptic equation

$$
-\operatorname{div}\left(A(x)\left(\operatorname{grad} z+\boldsymbol{p}-\boldsymbol{p}_{d}\right)\right)+a_{0} z=y-y_{d}, \quad x \in \Omega,
$$

which can be written in the form of the first order system

$$
\operatorname{div} \boldsymbol{q}+a_{0} z=y-y_{d}, \quad \boldsymbol{q}=-A(x)\left(\operatorname{grad} z+\boldsymbol{p}-\boldsymbol{p}_{d}\right), \quad x \in \Omega,
$$

and the boundary condition

$$
z=0, \quad x \in \partial \Omega \text {. }
$$

Next, we recall a result from Grisvard [10].

Lemma 2.1 ([10]). For every $p\left(2 \leq p<p_{\Omega}\right)$ and every function $\psi \in L^{p}(\Omega)$, the solution $\phi$ of

$$
-\operatorname{div}(A \operatorname{grad} \phi)+a_{0} \phi=\psi \quad \text { in } \Omega,\left.\phi\right|_{\partial \Omega}=0,
$$

belongs to $H_{0}^{1}(\Omega) \cap W^{2, p}(\Omega)$, where the constant $p_{\Omega}>2$ depending on the biggest interior angle of $\Omega$ and $A$. Moreover, there exists a positive constant $C$, independent of $a_{0}$ such that

$$
\|\phi\|_{W^{2, p}(\Omega)} \leq C\|\psi\|_{L^{p}(\Omega)} .
$$

Due to the above lemma, we know that the state equation and the adjoint equation admit unique solution in $H_{0}^{1}(\Omega) \cap W^{2, p}(\Omega)$, if $f, u, y_{d} \in L^{p}(\Omega)$ and $\boldsymbol{p}_{d} \in\left(W^{1, p}(\Omega)\right)^{2}$.

Let

$$
\boldsymbol{V}=H(\operatorname{div} ; \Omega)=\left\{\boldsymbol{v} \in\left(L^{2}(\Omega)\right)^{2}, \operatorname{div} \boldsymbol{v} \in L^{2}(\Omega)\right\}, \quad W=L^{2}(\Omega) .
$$

We recast (1.1)-(1.4) as the following weak form: find $(\boldsymbol{p}, y, u) \in \boldsymbol{V} \times W \times U_{a d}$ such that

$$
\begin{aligned}
& \min _{u \in U_{a d}}\left\{\frac{1}{2}\left\|\boldsymbol{p}-\boldsymbol{p}_{d}\right\|^{2}+\frac{1}{2}\left\|y-y_{d}\right\|^{2}+\frac{\nu}{2}\|u\|^{2}\right\}, \\
& \left(A^{-1} \boldsymbol{p}, \boldsymbol{v}\right)-(y, \operatorname{div} \boldsymbol{v})=0, \forall \boldsymbol{v} \in \boldsymbol{V}, \\
& (\operatorname{div} \boldsymbol{p}, w)+\left(a_{0} y, w\right)=(f+u, w), \forall w \in W .
\end{aligned}
$$

It follows from [16] that the optimal control problem (2.7)-(2.9) has a unique solution $(\boldsymbol{p}, y, u)$, and that a triplet $(\boldsymbol{p}, y, u)$ is the solution of (2.7)-(2.9) if and only if there is a co-state $(\boldsymbol{q}, z) \in \boldsymbol{V} \times W$ such that $(\boldsymbol{p}, y, \boldsymbol{q}, z, u)$ satisfies the following optimality conditions:

$$
\begin{aligned}
\left(A^{-1} \boldsymbol{p}, \boldsymbol{v}\right)-(y, \operatorname{div} \boldsymbol{v}) & =0, \forall \boldsymbol{v} \in \boldsymbol{V}, \\
(\operatorname{div} \boldsymbol{p}, w)+\left(a_{0} y, w\right) & =(f+u, w), \forall w \in W \\
\left(A^{-1} \boldsymbol{q}, \boldsymbol{v}\right)-(z, \operatorname{div} \boldsymbol{v}) & =-\left(\boldsymbol{p}-\boldsymbol{p}_{d}, \boldsymbol{v}\right), \forall \boldsymbol{v} \in \boldsymbol{V}, \\
(\operatorname{div} \boldsymbol{q}, w)+\left(a_{0} z, w\right) & =\left(y-y_{d}, w\right), \forall w \in W,
\end{aligned}
$$




$$
(\nu u+z, \tilde{u}-u) \geq 0, \forall \tilde{u} \in U_{a d},
$$

where $(\cdot, \cdot)$ is the inner product of $L^{2}(\Omega)$.

Introducing the projection

$$
\Pi_{[a, b]}(f(x))=\max \{a, \min (b, f(x))\},
$$

the optimal condition (2.14) can be expressed as follows:

$$
u(x)=\Pi_{[a, b]}\left(-\frac{1}{\nu} z(x)\right) .
$$

Let $\mathcal{T}_{h}$ denote a regular triangulation of the polygonal domain $\Omega, h_{T}$ denotes the diameter of $T$ and $h=\max h_{T}$. Let $\boldsymbol{V}_{h} \times W_{h} \subset \boldsymbol{V} \times W$ denotes the order $k=1$ Raviart-Thomas mixed finite element space [11, 21], namely,

$$
\forall T \in \mathcal{T}_{h}, \quad \boldsymbol{V}(T)=\boldsymbol{P}_{1}(T) \oplus \operatorname{span}\left(x P_{1}(T)\right), \quad W(T)=P_{1}(T),
$$

where $P_{1}(T)$ denote polynomials of total degree at most $1, \boldsymbol{P}_{1}(T)=\left(P_{1}(T)\right)^{2}$, $x=\left(x_{1}, x_{2}\right)$, which is treated as a vector, and

$$
\begin{aligned}
& V_{h}:=\left\{\boldsymbol{v}_{h} \in \boldsymbol{V}: \forall T \in \mathcal{T}_{h},\left.\boldsymbol{v}_{h}\right|_{T} \in \boldsymbol{V}(T)\right\}, \\
& W_{h}:=\left\{w_{h} \in W: \forall T \in \mathcal{T}_{h},\left.w_{h}\right|_{T} \in W(T)\right\}, \\
& U_{h}:=\left\{\tilde{u}_{h} \in U_{a d}: \forall T \in \mathcal{T}_{h},\left.\tilde{u}_{h}\right|_{T} \in W(T)\right\} .
\end{aligned}
$$

Before the mixed finite element scheme is given, we introduce two operators. Firstly, we define the standard $L^{2}(\Omega)$-projection [11] $P_{h}: W \rightarrow W_{h}$, which satisfies: for any $\phi \in W$

$$
\begin{aligned}
& \left(P_{h} \phi-\phi, w_{h}\right)=0, \quad \forall w_{h} \in W_{h}, \\
& \left\|\phi-P_{h} \phi\right\|_{0, \rho} \leq C h^{r}\|\phi\|_{r, \rho}, 1 \leq \rho \leq \infty, \forall \phi \in W^{r, \rho}(\Omega), \quad r=1,2 .
\end{aligned}
$$

Next, recall the Fortin projection (see [3] and [11]) $\Pi_{h}: \boldsymbol{V} \rightarrow \boldsymbol{V}_{h}$, which satisfies: for any $\boldsymbol{q} \in \boldsymbol{V}$

$$
\begin{aligned}
& \left(\operatorname{div}\left(\Pi_{h} \boldsymbol{q}-\boldsymbol{q}\right), w_{h}\right)=0, \quad \forall w_{h} \in W_{h}, \\
& \left\|\boldsymbol{q}-\Pi_{h} \boldsymbol{q}\right\| \leq C h^{r}\|\boldsymbol{q}\|_{r}, \quad \forall \boldsymbol{q} \in\left(H^{r}(\Omega)\right)^{2}, \quad r=1,2, \\
& \left\|\operatorname{div}\left(\boldsymbol{q}-\Pi_{h} \boldsymbol{q}\right)\right\| \leq C h^{r}\|\operatorname{div} \boldsymbol{q}\|_{r}, \quad \forall \operatorname{div} \boldsymbol{q} \in H^{r}(\Omega), \quad r=1,2 .
\end{aligned}
$$

We have the commuting diagram property

$$
\operatorname{div} \circ \Pi_{h}=P_{h} \circ \operatorname{div}: \boldsymbol{V} \rightarrow W_{h} \quad \text { and } \quad \operatorname{div}\left(I-\Pi_{h}\right) \boldsymbol{V} \perp W_{h},
$$

where and after, $I$ denote identity matrix.

Then the mixed finite element discretization of (2.7)-(2.9) is as follows: find $\left(\boldsymbol{p}_{h}, y_{h}, u_{h}\right) \in \boldsymbol{V}_{h} \times W_{h} \times U_{h}$ such that

$$
\begin{aligned}
& \min _{u_{h} \in U_{h}}\left\{\frac{1}{2}\left\|\boldsymbol{p}_{h}-\boldsymbol{p}_{d}\right\|^{2}+\frac{1}{2}\left\|y_{h}-y_{d}\right\|^{2}+\frac{\nu}{2}\left\|u_{h}\right\|^{2}\right\}, \\
& \left(A^{-1} \boldsymbol{p}_{h}, \boldsymbol{v}_{h}\right)-\left(y_{h}, \operatorname{div} \boldsymbol{v}_{h}\right)=0, \forall \boldsymbol{v}_{h} \in \boldsymbol{V}_{h}, \\
& \left(\operatorname{div} \boldsymbol{p}_{h}, w_{h}\right)+\left(a_{0} y_{h}, w_{h}\right)=\left(f+u_{h}, w_{h}\right), \forall w_{h} \in W_{h} .
\end{aligned}
$$


The optimal control problem (2.26)-(2.28) again has a unique solution $\left(\boldsymbol{p}_{h}, y_{h}\right.$, $\left.u_{h}\right)$, and that a triplet $\left(\boldsymbol{p}_{h}, y_{h}, u_{h}\right)$ is the solution of $(2.26)-(2.28)$ if and only if there is a co-state $\left(\boldsymbol{q}_{h}, z_{h}\right) \in \boldsymbol{V}_{h} \times W_{h}$ such that $\left(\boldsymbol{p}_{h}, y_{h}, \boldsymbol{q}_{h}, z_{h}, u_{h}\right)$ satisfies the following optimality conditions:

$$
\begin{aligned}
\left(A^{-1} \boldsymbol{p}_{h}, \boldsymbol{v}_{h}\right)-\left(y_{h}, \operatorname{div} \boldsymbol{v}_{h}\right) & =0, \forall \boldsymbol{v}_{h} \in \boldsymbol{V}_{h}, \\
\left(\operatorname{div} \boldsymbol{p}_{h}, w_{h}\right)+\left(a_{0} y_{h}, w_{h}\right) & =\left(f+u_{h}, w_{h}\right), \forall w_{h} \in W_{h}, \\
\left(A^{-1} \boldsymbol{q}_{h}, \boldsymbol{v}_{h}\right)-\left(z_{h}, \operatorname{div} \boldsymbol{v}_{h}\right) & =-\left(\boldsymbol{p}_{h}-\boldsymbol{p}_{d}, \boldsymbol{v}_{h}\right), \forall \boldsymbol{v}_{h} \in \boldsymbol{V}_{h}, \\
\left(\operatorname{div} \boldsymbol{q}_{h}, w_{h}\right)+\left(a_{0} z_{h}, w_{h}\right) & =\left(y_{h}-y_{d}, w_{h}\right), \forall w_{h} \in W_{h}, \\
\left(\nu u_{h}+z_{h}, \tilde{u}_{h}-u_{h}\right) & \geq 0, \forall \tilde{u}_{h} \in U_{h} .
\end{aligned}
$$

In the rest of the paper, we shall use some intermediate variables. For any control function $\tilde{u} \in U_{a d}$, we first define the state solution $(\boldsymbol{p}(\tilde{u}), y(\tilde{u}), \boldsymbol{q}(\tilde{u})$, $z(\tilde{u})) \in(\boldsymbol{V} \times W)^{2}$ associated with $\tilde{u}$ that satisfies

$$
\begin{aligned}
\left(A^{-1} \boldsymbol{p}(\tilde{u}), \boldsymbol{v}\right)-(y(\tilde{u}), \operatorname{div} \boldsymbol{v}) & =0, \quad \forall \boldsymbol{v} \in \boldsymbol{V}, \\
(\operatorname{div} \boldsymbol{p}(\tilde{u}), w)+\left(a_{0} y(\tilde{u}), w\right) & =(f+\tilde{u}, w), \quad \forall w \in W, \\
\left(A^{-1} \boldsymbol{q}(\tilde{u}), \boldsymbol{v}\right)-(z(\tilde{u}), \operatorname{div} \boldsymbol{v}) & =-\left(\boldsymbol{p}(\tilde{u})-\boldsymbol{p}_{d}, \boldsymbol{v}\right), \quad \forall \boldsymbol{v} \in \boldsymbol{V}, \\
(\operatorname{div} \boldsymbol{q}(\tilde{u}), w)+\left(a_{0} z(\tilde{u}), w\right) & =\left(y(\tilde{u})-y_{d}, w\right), \quad \forall w \in W .
\end{aligned}
$$

Then, we define the discrete state solution $\left(\boldsymbol{p}_{h}(\tilde{u}), y_{h}(\tilde{u}), \boldsymbol{q}_{h}(\tilde{u}), z_{h}(\tilde{u})\right) \in$ $\left(\boldsymbol{V}_{h} \times W_{h}\right)^{2}$ associated with $\tilde{u}$ that satisfies

$$
\begin{aligned}
\left(A^{-1} \boldsymbol{p}_{h}(\tilde{u}), \boldsymbol{v}_{h}\right)-\left(y_{h}(\tilde{u}), \operatorname{div} \boldsymbol{v}_{h}\right) & =0, \quad \forall \boldsymbol{v}_{h} \in \boldsymbol{V}_{h}, \\
\left(\operatorname{div} \boldsymbol{p}_{h}(\tilde{u}), w_{h}\right)+\left(a_{0} y_{h}(\tilde{u}), w_{h}\right) & =\left(f+\tilde{u}, w_{h}\right), \quad \forall w_{h} \in W_{h}, \\
\left(A^{-1} \boldsymbol{q}_{h}(\tilde{u}), \boldsymbol{v}_{h}\right)-\left(z_{h}(\tilde{u}), \operatorname{div} \boldsymbol{v}_{h}\right) & =-\left(\boldsymbol{p}_{h}(\tilde{u})-\boldsymbol{p}_{d}, \boldsymbol{v}_{h}\right), \quad \forall \boldsymbol{v}_{h} \in \boldsymbol{V}_{h}, \\
\left(\operatorname{div} \boldsymbol{q}_{h}(\tilde{u}), w_{h}\right)+\left(a_{0} z_{h}(\tilde{u}), w_{h}\right) & =\left(y_{h}(\tilde{u})-y_{d}, w_{h}\right), \quad \forall w_{h} \in W_{h} .
\end{aligned}
$$

Thus, as we defined, the exact solution and its approximation can be written in the following way:

$$
\begin{aligned}
(\boldsymbol{p}, y, \boldsymbol{q}, z) & =(\boldsymbol{p}(u), y(u), \boldsymbol{q}(u), z(u)), \\
\left(\boldsymbol{p}_{h}, y_{h}, \boldsymbol{q}_{h}, z_{h}\right) & =\left(\boldsymbol{p}_{h}\left(u_{h}\right), y_{h}\left(u_{h}\right), \boldsymbol{q}_{h}\left(u_{h}\right), z_{h}\left(u_{h}\right)\right) .
\end{aligned}
$$

\section{3. $L^{\infty}$-error estimates}

In this section, we will give a detailed $L^{\infty}$-error analysis. Firstly, by use of the duality argument, we can easily get the following result.

Lemma 3.1. For any $\tilde{u} \in U_{a d}$, let $(\boldsymbol{p}(\tilde{u}), y(\tilde{u}), \boldsymbol{q}(\tilde{u}), z(\tilde{u})) \in(\boldsymbol{V} \times W)^{2}$ be the solution of (2.34)-(2.37) and $\left(\boldsymbol{p}_{h}(\tilde{u}), y_{h}(\tilde{u}), \boldsymbol{q}_{h}(\tilde{u}), z_{h}(\tilde{u})\right) \in\left(\boldsymbol{V}_{h} \times W_{h}\right)^{2}$ be the solution of (2.38)-(2.41), respectively. If the intermediate solution satisfies

$$
\boldsymbol{p}(\tilde{u}), \boldsymbol{q}(\tilde{u}) \in\left(H^{1}(\Omega)\right)^{2},
$$

then we have

$$
\left\|y(\tilde{u})-y_{h}(\tilde{u})\right\| \leq C h^{2}
$$




$$
\left\|z(\tilde{u})-z_{h}(\tilde{u})\right\| \leq C h^{2} .
$$

By using the stability result of mixed finite element methods [3], we have the following results.

Lemma 3.2. Let $\left(\boldsymbol{p}_{h}(u), y_{h}(u), \boldsymbol{q}_{h}(u), z_{h}(u)\right) \in\left(\boldsymbol{V}_{h} \times W_{h}\right)^{2}$ and $\left(\boldsymbol{p}_{h}, y_{h}, \boldsymbol{q}_{h}, z_{h}\right)$ $\in\left(\boldsymbol{V}_{h} \times W_{h}\right)^{2}$ be the solutions of (2.34)-(2.37) with $\tilde{u}=u$ and $\tilde{u}=u_{h}$, respectively. Then we have

$$
\begin{aligned}
& \left\|y_{h}(u)-y_{h}\right\|+\left\|\boldsymbol{p}_{h}(u)-\boldsymbol{p}_{h}\right\| \leq C\left\|u-u_{h}\right\|, \\
& \left\|z_{h}(u)-z_{h}\right\|+\left\|\boldsymbol{q}_{h}(u)-\boldsymbol{q}_{h}\right\| \leq C\left\|u-u_{h}\right\| .
\end{aligned}
$$

Let

$$
\begin{aligned}
& \Omega^{+}=\left\{\bigcup T: T \subset \Omega, a<\left.u(x)\right|_{T}<b\right\}, \\
& \Omega^{0}=\left\{\bigcup T: T \subset \Omega,\left.u(x)\right|_{T} \equiv a, \text { or }\left.u(x)\right|_{T} \equiv b\right\}, \\
& \Omega^{b}=\Omega \backslash\left(\Omega^{+} \cup \Omega^{0}\right) .
\end{aligned}
$$

It is easy to check that the three parts do not intersect on each other, and $\Omega=\Omega^{+} \cup \Omega^{0} \cup \Omega^{b}$. In this paper we assume that $u$ and $\mathcal{T}_{h}$ are regular such that meas $\left(\Omega^{b}\right) \leq C h$ (see $\left.[19]\right)$.

Theorem 3.1. Let $(\boldsymbol{p}, y, \boldsymbol{q}, z, u) \in(\boldsymbol{V} \times W)^{2} \times U$ and $\left(\boldsymbol{p}_{h}, y_{h}, \boldsymbol{q}_{h}, z_{h}, u_{h}\right) \in$ $\left(\boldsymbol{V}_{h} \times W_{h}\right)^{2} \times U_{h}$ be the solutions of (2.6)-(2.10) and (2.18)-(2.22). Assume that $u \in W^{1, \infty}(\Omega)$ and $u \in H^{2}\left(\Omega^{+}\right)$. Then we have

$$
\begin{aligned}
& \left\|u-u_{h}\right\| \leq C h^{\frac{3}{2}}, \\
& \left\|y-y_{h}\right\| \leq C h^{\frac{3}{2}}, \\
& \left\|z-z_{h}\right\| \leq C h^{\frac{3}{2}} .
\end{aligned}
$$

Proof. First, let $u_{I} \in W_{h}$ be the standard lagrange interpolation of $u$ such that $u_{I}(p)=u(p)$ for all vertices $p$. Then it is easy to see that $u_{I} \in U_{h}$. Note that $u \in W^{1, \infty}(\Omega)$ and $u \in H^{2}\left(\Omega^{+}\right)$. We have

$$
\left\|u-u_{I}\right\|_{0, \Omega^{+}} \leq C h^{2}\|u\|_{2, \Omega^{+}}, \quad\left\|u-u_{I}\right\|_{0, \infty, \Omega^{b}} \leq C h\|u\|_{1, \infty, \Omega^{b}},
$$

and hence,

$$
\begin{aligned}
\left\|u-u_{I}\right\|^{2} & =\int_{\Omega}\left(u-u_{I}\right)^{2} \\
& =\int_{\Omega^{+}}\left(u-u_{I}\right)^{2}+\int_{\Omega^{0}}\left(u-u_{I}\right)^{2}+\int_{\Omega^{b}}\left(u-u_{I}\right)^{2} \\
& \leq C h^{4}\|u\|_{2, \Omega^{+}}^{2}+0+C h^{2}\|u\|_{1, \infty, \Omega^{b}}^{2} \operatorname{meas}\left(\Omega^{b}\right) \\
& \leq C h^{4}\|u\|_{2, \Omega^{+}}^{2}+C h^{3}\|u\|_{1, \infty, \Omega^{b}}^{2} \\
& \leq C h^{3}\left(\|u\|_{2, \Omega^{+}}^{2}+\|u\|_{1, \infty}^{2}\right) \leq C h^{3} .
\end{aligned}
$$


By taking $\tilde{u}=u_{h}$ in (2.14) and $\tilde{u}_{h}=u_{I}$ in (2.33) we have that

$$
\left(\nu u+z, u_{h}-u\right) \geq 0
$$

and

$$
\left(\nu u_{h}+z_{h}, u_{I}-u_{h}\right) \geq 0 .
$$

Note that $u_{I}-u_{h}=u_{I}-u+u-u_{h}$ in (3.11) and add the inequalities (3.10) and (3.11), we have

$$
\left(\nu u_{h}+z_{h}-\nu u-z, u-u_{h}\right)+\left(\nu u_{h}+z_{h}, u_{I}-u\right) \geq 0 .
$$

By using (3.12), we have

$$
\begin{aligned}
\nu\left\|u-u_{h}\right\|^{2}= & \left(\nu u-\nu u_{h}, u-u_{h}\right) \\
\leq & \left(z_{h}-z, u-u_{h}\right)+\left(\nu u_{h}+z_{h}, u_{I}-u\right) \\
= & \left(z_{h}-z_{h}(u), u-u_{h}\right)+\left(z_{h}(u)-z, u-u_{h}\right) \\
& +\left(\nu u_{h}-\nu u, u_{I}-u\right)+\left(z_{h}-z_{h}(u), u_{I}-u\right) \\
& +\left(z_{h}(u)-z, u_{I}-u\right)+\left(\nu u+z, u_{I}-u\right) .
\end{aligned}
$$

Now, we estimate all terms on the right side of (3.13). From (2.38)-(2.41), we derive the following error equations

$$
\begin{aligned}
&\left(A^{-1}\left(\boldsymbol{p}_{h}(u)-\boldsymbol{p}_{h}\right), \boldsymbol{v}_{h}\right)-\left(y_{h}(u)-y_{h}, \operatorname{div} \boldsymbol{v}_{h}\right)=0, \quad \forall \boldsymbol{v}_{h} \in \boldsymbol{V}_{h}, \\
&\left(\operatorname{div}\left(\boldsymbol{p}_{h}(u)-\boldsymbol{p}_{h}\right), w_{h}\right)+\left(a_{0}\left(y_{h}(u)-y_{h}\right), w_{h}\right) \\
&=\left(u-u_{h}, w_{h}\right), \quad \forall w_{h} \in W_{h} \\
&\left(A^{-1}\left(\boldsymbol{q}_{h}(u)-\boldsymbol{q}_{h}\right), \boldsymbol{v}_{h}\right)-\left(z_{h}(u)-z_{h}, \operatorname{div} \boldsymbol{v}_{h}\right) \\
&=-\left(\boldsymbol{p}_{h}(u)-\boldsymbol{p}_{h}, \boldsymbol{v}_{h}\right), \forall \boldsymbol{v}_{h} \in \boldsymbol{V}_{h},
\end{aligned}
$$

$$
\begin{aligned}
& \left(\operatorname{div}\left(\boldsymbol{q}_{h}(u)-\boldsymbol{q}_{h}\right), w_{h}\right)+\left(a_{0}\left(z_{h}(u)-z_{h}, w_{h}\right)\right. \\
= & \left(y_{h}(u)-y_{h}, w_{h}\right), \quad \forall w_{h} \in W_{h} .
\end{aligned}
$$

Set $\boldsymbol{v}_{h}=\boldsymbol{q}_{h}(u)-\boldsymbol{q}_{h}$ in $(3.14), w_{h}=z_{h}(u)-z_{h}$ in $(3.15), \boldsymbol{v}_{h}=\boldsymbol{p}_{h}(u)-\boldsymbol{p}_{h}$ in (3.16) and $w_{h}=y_{h}(u)-y_{h}$ in (3.17), respectively. It is easy to see that

$$
\left(z_{h}-z_{h}(u), u-u_{h}\right)=-\left\|y_{h}(u)-y_{h}\right\|^{2}-\left\|\boldsymbol{p}_{h}(u)-\boldsymbol{p}_{h}\right\|^{2} \leq 0 .
$$

It follows from Lemma 3.1 that

$$
\left(z_{h}(u)-z, u-u_{h}\right) \leq C\left\|z_{h}(u)-z\right\| \cdot\left\|u-u_{h}\right\| \leq C h^{2}\left\|u-u_{h}\right\| .
$$

By Lemma 3.1, Lemma 3.2, the Cauchy inequality and the approximation property (3.9) of the interpolation $u_{I}$, we get

$$
\begin{array}{r}
\left(\nu u_{h}-\nu u, u_{I}-u\right) \leq C\left\|u_{h}-u\right\| \cdot\left\|u_{I}-u\right\| \leq C h^{\frac{3}{2}}\left\|u-u_{h}\right\|, \\
\left(z_{h}-z_{h}(u), u_{I}-u\right) \leq C\left\|z_{h}-z_{h}(u)\right\| \cdot\left\|u_{I}-u\right\| \leq C h^{\frac{3}{2}}\left\|u-u_{h}\right\|
\end{array}
$$


and

$$
\left(z_{h}(u)-z, u_{I}-u\right) \leq C\left\|z_{h}(u)-z\right\| \cdot\left\|u-u_{I}\right\| \leq C h^{\frac{7}{2}} .
$$

Moreover, it follows from (2.14) that $\nu u+z=0$ on $\Omega^{+}$. It is easy to see that $u-u_{I}=0$ on $\Omega^{0}$. Note that for all element $T^{b} \subset \Omega^{b}$, there is $x_{0} \in T^{b}$ such that $a<u\left(x_{0}\right)<b$, and hence $(\nu u+z)\left(x_{0}\right)=0$. Then

$$
\|\nu u+z\|_{0, \infty, T^{b}}=\left\|\nu u+z-(\nu u+z)\left(x_{0}\right)\right\|_{0, \infty, T^{b}} \leq C h\|\nu u+z\|_{1, \infty, T^{b}} .
$$

Thus

$$
\begin{aligned}
\left(\nu u+z, u_{I}-u\right)= & \int_{\Omega^{+}}(\nu u+z)\left(u_{I}-u\right)+\int_{\Omega^{0}}(\nu u+z)\left(u_{I}-u\right) \\
& +\int_{\Omega^{b}}(\nu u+z)\left(u_{I}-u\right) \\
= & 0+0+\int_{\Omega^{b}}(\nu u+z)\left(u_{I}-u\right) \\
\leq & \|\nu u+z\|_{0, \infty, \Omega^{b}}\left\|u_{I}-u\right\|_{0, \infty, \Omega^{b}} \operatorname{meas}\left(\Omega^{b}\right) \\
\leq & C h^{2}\|\nu u+z\|_{1, \infty, \Omega^{b}}\|u\|_{1, \infty, \Omega^{b}} \operatorname{meas}\left(\Omega^{b}\right) \\
\leq & C h^{3} .
\end{aligned}
$$

Substituting the estimates (3.18)-(3.23) in (3.13), we derive the result (3.5). By use of Lemmas 3.1-3.2 and (3.5), we obtain

$$
\begin{aligned}
& \left\|y-y_{h}\right\| \leq\left\|y-y_{h}(u)\right\|+\left\|y_{h}(u)-y_{h}\right\| \leq C\left\|u-u_{h}\right\|+C h^{2} \leq C h^{\frac{3}{2}}, \\
& \left\|z-z_{h}\right\| \leq\left\|z-z_{h}(u)\right\|+\left\|z_{h}(u)-z_{h}\right\| \leq C\left\|u-u_{h}\right\|+C h^{2} \leq C h^{\frac{3}{2}} .
\end{aligned}
$$

Thus the estimates (3.6)-(3.7) are proved.

Lemma 3.3. Assume that all the conditions in Theorem 3.1 are valid. Let $(y, z)$ be the solution of (2.10)-(2.14) and $\left(y_{h}, z_{h}\right)$ be the solution of (2.29)(2.33), respectively. Then we have

$$
\left\|y-y_{h}\right\|_{0, \infty}+\left\|z-z_{h}\right\|_{0, \infty} \leq c h,
$$

where $c$ is a positive constant.

Proof. Using (2.21) and Lemma 3.1, we can derive

$$
\left\|P_{h} y\left(u_{h}\right)-y_{h}\right\|+\left\|P_{h} z\left(u_{h}\right)-z_{h}\right\| \leq C h^{2} .
$$

From equations (1.2) and (2.1), we have the following error equations

$$
-\operatorname{div}\left(A \operatorname{grad}\left(y-y\left(u_{h}\right)\right)\right)+a_{0}\left(y-y\left(u_{h}\right)\right)=u-u_{h}
$$

and

$$
\begin{aligned}
& -\operatorname{div}\left(A \operatorname{grad}\left(z-z\left(u_{h}\right)\right)\right)+a_{0}\left(z-z\left(u_{h}\right)\right) \\
= & -\operatorname{div}\left(A^{2} \operatorname{grad}\left(y-y\left(u_{h}\right)\right)\right)+y-y\left(u_{h}\right) .
\end{aligned}
$$


Using (2.5), Theorem 3.1 and the classical imbedding theorem, we have

$$
\begin{aligned}
\left\|y-y\left(u_{h}\right)\right\|_{0, \infty} & =\left\|y-y\left(u_{h}\right)\right\|_{C(\bar{\Omega})} \\
& \leq C\left\|y-y\left(u_{h}\right)\right\|_{2} \\
& \leq C\left\|u-u_{h}\right\| \\
& \leq C h^{\frac{3}{2}}
\end{aligned}
$$

and

$$
\begin{aligned}
\left\|z-z\left(u_{h}\right)\right\|_{0, \infty} & =\left\|z-z\left(u_{h}\right)\right\|_{C(\bar{\Omega})} \\
& \leq C\left\|z-z\left(u_{h}\right)\right\|_{2} \\
& \leq C\left(\left\|\operatorname{div}\left(A^{2} \operatorname{grad}\left(y-y\left(u_{h}\right)\right)\right)\right\|+\left\|y-y\left(u_{h}\right)\right\|\right) \\
& \leq C\left\|y-y\left(u_{h}\right)\right\|_{2} \\
& \leq C\left\|u-u_{h}\right\| \\
& \leq C h^{\frac{3}{2}}
\end{aligned}
$$

Thus, by (2.21), (3.27), (3.30), (3.31) and the inverse estimate, we have

$$
\begin{aligned}
& \left\|y-y_{h}\right\|_{0, \infty}+\left\|z-z_{h}\right\|_{0, \infty} \\
\leq & \left\|y-y\left(u_{h}\right)\right\|_{0, \infty}+\left\|z-z\left(u_{h}\right)\right\|_{0, \infty} \\
& +\left\|y\left(u_{h}\right)-P_{h} y\left(u_{h}\right)\right\|_{0, \infty}+\left\|z\left(u_{h}\right)-P_{h} z\left(u_{h}\right)\right\|_{0, \infty} \\
& +\left\|P_{h} y\left(u_{h}\right)-y_{h}\right\|_{0, \infty}+\left\|P_{h} z\left(u_{h}\right)-z_{h}\right\|_{0, \infty} \\
\leq & C h^{\frac{3}{2}}+C h\left(\left\|y\left(u_{h}\right)\right\|_{1, \infty}+\left\|z\left(u_{h}\right)\right\|_{1, \infty}\right) \\
& +C h^{-1}\left(\left\|P_{h} y\left(u_{h}\right)-y_{h}\right\|+\left\|P_{h} z\left(u_{h}\right)-z_{h}\right\|\right) \\
\leq & c h .
\end{aligned}
$$

We complete the proof.

Next, we introduce a new notation for piecewise linear functions. Let $E_{j}$ be an arbitrary vertex of a triangle $T_{i}$. Then, we define a linear function $\tilde{e}_{i, j}$ on $T_{i}$ by

$$
\tilde{e}_{i, j}\left(E_{k}\right)=\delta_{j k},
$$

where $\delta_{j k}$ is the Kronecker symbol and $E_{k}$ is a vertex of $T_{i}$. Next, we introduce our basis function

$$
e_{i j}=- \begin{cases}\tilde{e}_{i, j} & \text { on } T_{i} \\ 0 & \text { else. }\end{cases}
$$

Thus, we can represent the functions $u_{h}$ and $z_{h}$ by

$$
u_{h}(x)=\sum_{T_{i}} \sum_{j=1}^{3} u_{i j} e_{i j}(x)
$$


and

$$
z_{h}(x)=\sum_{T_{i}} \sum_{j=1}^{3} z_{i j} e_{i j}(x),
$$

where $u_{i j}=\lim _{x \rightarrow E_{j}, x \in T_{i}} u_{h}(x)$ and $z_{i j}=\lim _{x \rightarrow E_{j}, x \in T_{i}} z_{h}(x)$.

Let $T_{i}$ be an arbitrary triangle and $E_{j}$ an arbitrary vertex. We denote the set of all vertices of $T_{i}$ excepted $E_{j}$ by $N_{i}\left(E_{j}\right)$.

Lemma 3.4. For every triangle $T_{i}$ and every indices $k, j$ with $E_{k} \in N_{i}\left(E_{j}\right)$ it holds

$$
\frac{1}{\nu}\left|z_{i j}-z_{i k}\right| \leq D h
$$

with

$$
D=\frac{L+2 c}{\nu}
$$

where $L$ denotes the Lipschitz constant of $z$.

Proof. Because of Lemma 2.1, $z$ belongs to $W^{2, p}(\Omega)$ for a certain $p>2$. Therefore $z$ is Lipschitz continuous and we have

$$
\left|z\left(E_{j}\right)-z\left(E_{k}\right)\right| \leq L h .
$$

Combining this inequality with (3.26), we obtain

$$
\begin{aligned}
\left|z_{i j}-z_{i k}\right| & \leq\left|z_{i j}-z\left(E_{j}\right)\right|+\left|z\left(E_{j}\right)-z\left(E_{k}\right)\right|+\left|z\left(E_{k}\right)-z_{i k}\right| \\
& \leq c h+L h+c h .
\end{aligned}
$$

Dividing by $\nu$, we obtain (3.36).

Lemma 3.5. For a fixed triangle $T_{i}$ and arbitrary basis functions $e_{i j}, e_{i k}(j \neq$ $k)$, we have

$$
\left(e_{i j}, e_{i j}\right)=2\left(e_{i j}, e_{i k}\right) \text {. }
$$

Proof. The element mass matrix of the reference element $T_{r}$ is given by

$$
M_{r}=\left(\left(e_{i j}, e_{i k}\right)\right)_{j, k=1,2,3}=\frac{1}{24}\left(\begin{array}{ccc}
2 & 1 & 1 \\
1 & 2 & 1 \\
1 & 1 & 2
\end{array}\right) \text {. }
$$

Clearly, the entries of this matrix have the desired property. The mass matrix of an arbitrary triangle $T_{s}$ is given by

$$
M_{s}=\frac{\left|T_{s}\right|}{\left|T_{r}\right|} M_{r} .
$$

Multiplication with a scalar factor preserves this property. 
In order to derive the $L^{\infty}$-error estimates for the control variable, we need to analysis the following quantity

$$
M:=\max _{i j}\left|u_{i j}-\Pi_{[a, b]}\left(-\frac{1}{\nu} z_{i j}\right)\right| .
$$

In all what follows, the index $i j$ denotes a fixed vertex $E_{j}$ and a corresponding triangle $T_{i}$ where this maximum is attained.

Equation (3.42) means that one of the following two cases occurs

$$
\begin{aligned}
\text { (I) } \quad M & =u_{i j}-\Pi_{[a, b]}\left(-\frac{1}{\nu} z_{i j}\right), \\
\text { (II) } \quad M & =-\left(u_{i j}-\Pi_{[a, b]}\left(-\frac{1}{\nu} z_{i j}\right)\right) .
\end{aligned}
$$

Lemma 3.6. Assume that $M>0$. Then, the control $\tilde{u}_{h}=u_{h}-\varepsilon e_{i j}$ is admissible in the case (3.43) and $\tilde{u}_{h}=u_{h}+\varepsilon e_{i j}$ is admissible in the case (3.44) for a sufficiently small $\varepsilon>0$. Moreover, the following inequalities hold true.

(3.45) $M=u_{i j}-\Pi_{[a, b]}\left(-\frac{1}{\nu} z_{i j}\right) \leq u_{i j}+\frac{1}{\nu} z_{i j}$ in the case $(\mathbf{I})$,

(3.46) $\quad M=-\left(u_{i j}-\Pi_{[a, b]}\left(-\frac{1}{\nu} z_{i j}\right)\right) \leq-\left(u_{i j}+\frac{1}{\nu} z_{i j}\right)$ in the case $(\mathbf{I I})$.

Proof. We discuss only case $(\mathbf{I})$, the case $(\mathbf{I I})$ can be obtained similarly. Since $M$ is positive, using the fact that $a \leq \Pi_{[a, b]}\left(\frac{1}{\nu} z_{i j}\right) \leq b$, we conclude that

$$
u_{i j}>\Pi_{[a, b]}\left(-\frac{1}{\nu} z_{i j}\right) \geq a .
$$

Consequently, there exists a $\varepsilon>0$ such that

$$
u_{i j}-\varepsilon>a .
$$

From (3.48), we can see that the control $\tilde{u}_{h}=u_{h}-\varepsilon e_{i j}$ is admissible. Again by use of $M>0$ and $u_{i j} \in[a, b]$ we have $\Pi_{[a, b]}\left(-\frac{1}{\nu} z_{i j}\right) \leq b$. Therefore, we have $\Pi_{[a, b]}\left(-\frac{1}{\nu} z_{i j}\right) \geq-\frac{1}{\nu} z_{i j}$ and consequently

$$
M=u_{i j}-\Pi_{[a, b]}\left(-\frac{1}{\nu} z_{i j}\right) \leq u_{i j}+\frac{1}{\nu} z_{i j},
$$

which implies (3.45).

Lemma 3.7. Let $\tilde{u}_{h}=u_{h}-\varepsilon e_{m l}$ and $v_{h}=u_{h}+\varepsilon e_{r s}$ be admissible for certain indices $l, m, r, s$ and $\varepsilon>0$. Then the following inequalities hold true.

$$
\begin{aligned}
& u_{m l}+\frac{1}{\nu} z_{m l} \leq-\frac{1}{2} \sum_{E_{k} \in N_{m}\left(E_{l}\right)}\left(u_{m k}+\frac{1}{\nu} z_{m k}\right), \\
& u_{r s}+\frac{1}{\nu} z_{r s} \geq-\frac{1}{2} \sum_{E_{k} \in N_{r}\left(E_{s}\right)}\left(u_{r k}+\frac{1}{\nu} z_{r k}\right) .
\end{aligned}
$$


Proof. We discuss only (3.50), (3.51) can be obtained similarly. Taking $\tilde{u}_{h}=$ $u_{h}-\varepsilon e_{m l}$ in (2.33), we arrive at

$$
\left(\nu u_{h}+z_{h},-\varepsilon e_{m l}\right) \geq 0 .
$$

Furthermore, (3.52) can be written as

$$
\left(\nu u_{m l}+z_{m l}\right)\left(e_{m l}, e_{m l}\right) \leq-\sum_{E_{k} \in N_{m}\left(E_{l}\right)}\left(\nu u_{m k}+z_{m k}\right)\left(e_{m l}, e_{m k}\right) .
$$

Using (3.39), we find

$$
\left(\nu u_{m l}+z_{m l}\right)\left(e_{m l}, e_{m l}\right) \leq-\frac{1}{2}\left(e_{m l}, e_{m l}\right) \sum_{E_{k} \in N_{m}\left(E_{l}\right)}\left(\nu u_{m k}+z_{m k}\right) .
$$

Division by $\left(e_{m l}, e_{m l}\right)$ yields $(3.50)$

Lemma 3.8. Let $M>0$ and $i j$ be an index where the maximum in (3.42) is attained. Then there exists an index $m$ with $E_{m} \in N_{i}\left(E_{j}\right)$ such that

$$
\begin{aligned}
& \Pi_{[a, b]}\left(-\frac{1}{\nu} z_{i m}\right) \leq-\frac{1}{\nu} z_{i m} \text { in the case }(\mathbf{I}), \\
& \Pi_{[a, b]}\left(-\frac{1}{\nu} z_{i m}\right) \geq-\frac{1}{\nu} z_{i m} \text { in the case }(\mathbf{I I}) .
\end{aligned}
$$

Proof. We discuss only (3.55), (3.56) can be obtained similarly. The discussion of (3.55) can be splitted in two partial cases:

Case I. There exist an index $l$ with $E_{l} \in N_{i}\left(E_{j}\right)$ such that

$$
\nu u_{i l}+z_{i l}>0 \text {. }
$$

We can apply (3.50) for the index $i j$, since $\tilde{u}_{h}=u_{h}-\varepsilon e_{i j}$ is admissible (Lemma 3.6). Then, we can increase the right-hand side of (3.50) by omitting the term $-\left(\nu u_{i l}+z_{i l}\right)$

$$
\left(\nu u_{i j}+z_{i j}\right)<-\frac{1}{2} \sum_{E_{k} \in N_{i}\left(E_{j}\right), E_{k} \neq E_{l}}\left(\nu u_{i k}+z_{i k}\right) .
$$

From (3.58), it is easy to see that

$$
\left(\nu u_{i j}+z_{i j}\right)<-\max _{E_{k} \in N_{i}\left(E_{j}\right)}\left(\nu u_{i k}+z_{i k}\right) .
$$

We denote an index, where this maximum is attained by $m$

$$
-\left(\nu u_{i m}+z_{i m}\right)=-\max _{E_{k} \in N_{i}\left(E_{j}\right)}\left(\nu u_{i k}+z_{i k}\right) .
$$

From (3.60) and (3.49), we find that

$$
M=u_{i j}-\Pi_{[a, b]}\left(-\frac{1}{\nu} z_{i j}\right) \leq u_{i j}+\frac{1}{\nu} z_{i j}<-\left(u_{i m}+\frac{1}{\nu} z_{i m}\right) .
$$


By definition of $M$, we have

$$
-\left(u_{i m}-\Pi_{[a, b]}\left(-\frac{1}{\nu} z_{i m}\right)\right) \leq M .
$$

Hence, we derive

$$
\Pi_{[a, b]}\left(-\frac{1}{\nu} z_{i m}\right) \leq-\frac{1}{\nu} z_{i m} .
$$

Case II. For all indices $l$ with $E_{l} \in N_{i}\left(E_{j}\right)$ we have

$$
\nu u_{i l}+z_{i l} \leq 0 \text {. }
$$

Using (3.50), we find that

$$
\left(\nu u_{i j}+z_{i j}\right) \leq-\frac{3}{2} \max _{E_{k} \in N_{i}\left(E_{j}\right)}\left(\nu u_{i k}+z_{i k}\right)
$$

Again, we denote an index, where this maximum is attained by $m$

$$
-\left(\nu u_{i m}+z_{i m}\right)=-\max _{E_{k} \in N_{i}\left(E_{j}\right)}\left(\nu u_{i k}+z_{i k}\right) .
$$

Next, we will show that $u_{i m}=b$. We assuming $u_{i m}<b$, the control $\tilde{u}_{h}=$ $u_{h}+\varepsilon e_{i m}$ is admissible for sufficiently small $\varepsilon$. Thus, we can apply (3.51) for the index $i m$

$$
-\left(u_{i m}+\frac{1}{\nu} z_{i m}\right) \leq \frac{1}{2} \sum_{E_{k} \in N_{i}\left(E_{m}\right)}\left(u_{i k}+\frac{1}{\nu} z_{i k}\right) .
$$

It follows from (3.64) and (3.67) that

$$
-\left(u_{i m}+\frac{1}{\nu} z_{i m}\right) \leq \frac{1}{2}\left(u_{i j}+\frac{1}{\nu} z_{i j}\right) .
$$

By (3.65), (3.66) and (3.68), we get

$$
u_{i j}+\frac{1}{\nu} z_{i j} \leq \frac{3}{4}\left(u_{i j}+\frac{1}{\nu} z_{i j}\right)
$$

or $u_{i j}+\frac{1}{\nu} z_{i j} \leq 0$. This is a contradiction to (3.49) and $M>0$. Consequently, we have $u_{i m}=b$. Using the inequalities $M>0,(3.49)$ and (3.65), we know that

$$
0<M \leq-\left(\nu u_{i m}+z_{i m}\right)=-\left(\nu b+z_{i m}\right),
$$

which yields to

$$
b<-\frac{1}{\nu} z_{i m}
$$

Thus, we complete the proof.

Lemma 3.9. Assume that $D h<b-a$, then we have the following estimate

$$
M=\max _{i j}\left|u_{i j}-\Pi_{[a, b]}\left(-\frac{1}{\nu} z_{i j}\right)\right|<D h .
$$


Proof. For $M=0,(3.72)$ is obvious. Let us assume $M>0$. Again, we discuss only the Case (I). From (3.55), we have

$$
b=\Pi_{[a, b]}\left(-\frac{1}{\nu} z_{i m}\right)<-\frac{1}{\nu} z_{i m} .
$$

From (3.73) and (3.36), we get

$$
-\frac{1}{\nu} z_{i j}>b-D h
$$

By assumption $D h<b-a$ and (3.74), we find that

$$
-\frac{1}{\nu} z_{i j}>a .
$$

From (I)

$$
u_{i j}-\Pi_{[a, b]}\left(-\frac{1}{\nu} z_{i j}\right)=M>0
$$

and $u_{i j} \leq b$ we obtain

$$
\Pi_{[a, b]}\left(-\frac{1}{\nu} z_{i j}\right)<b .
$$

It follows from (3.75) and (3.77) that

$$
-\frac{1}{\nu} z_{i j}=\Pi_{[a, b]}\left(-\frac{1}{\nu} z_{i j}\right)
$$

which implies

$$
u_{i j}+\frac{1}{\nu} z_{i j}=u_{i j}-\Pi_{[a, b]}\left(-\frac{1}{\nu} z_{i j}\right)=M .
$$

By use of $u_{i j} \leq b$ and $\frac{1}{\nu} z_{i j}<-(b-D h)$, we find that

$$
u_{i j}+\frac{1}{\nu} z_{i j}<b-(b-D h)=D h .
$$

Thus, (3.72) can be proved by (3.79) and (3.80).

Now, we can prove the $L^{\infty}$-error estimate for the control variable.

Theorem 3.2. Let $u$ be the solution of (2.10)-(2.14) and $u_{h}$ be the solution of (2.29)-(2.33), respectively. Assume that all the conditions in previous Lemmas and Theorem are valid. Then, we have

$$
\left\|u-u_{h}\right\|_{0, \infty} \leq C h
$$

where $C=D+(c+L) / \nu$. 
Proof. Because of $u_{h} \in[a, b]$, it is easy to see that (3.81) holds for $D h \geq b-a$. Now we consider the case $D h<b-a$. First, we derive a estimate for the grid points. From Lemma 3.9, we have

$$
\max _{i j}\left|u_{i j}-\Pi_{[a, b]}\left(-\frac{1}{\nu} z_{i j}\right)\right|<D h .
$$

From Lemma 3.3 and the Lipschitz continuity of projection operator we get

$$
\max _{i j}\left|\Pi_{[a, b]}\left(-\frac{1}{\nu} z\left(E_{j}\right)\right)-\Pi_{[a, b]}\left(-\frac{1}{\nu} z_{i j}\right)\right|<\frac{c}{\nu} h .
$$

From (2.14), we have

$$
u\left(E_{j}\right)=\Pi_{[a, b]}\left(-\frac{1}{\nu} z\left(E_{j}\right)\right) .
$$

Combining (3.82)-(3.84) with the triangle inequality, we derive

$$
\max _{i j}\left|u_{i j}-u\left(E_{j}\right)\right|<\left(D+\frac{c}{\nu}\right) h \text {. }
$$

Second, for a non grid point $x \in T_{i}$ we find a convex linear combination of the vertices $E_{j}$ of the corresponding triangle

$$
x=\sum_{E_{j} \in T_{i}} \lambda_{j} E_{j}, \quad \sum_{E_{j} \in T_{i}} \lambda_{j}=1 .
$$

Since $u_{h}$ is linear on $T_{i}$, we have

$$
\begin{aligned}
\left|u_{h}(x)-u(x)\right| & =\left|\sum_{E_{j} \in T_{i}} \lambda_{j} u_{i j}-u(x)\right| \\
& \leq \sum_{E_{j} \in T_{i}} \lambda_{j}\left|u_{i j}-u\left(E_{j}\right)\right|+\sum_{E_{j} \in T_{i}} \lambda_{j}\left|u\left(E_{j}\right)-u(x)\right| \\
& \leq\left(D+\frac{c}{\nu}\right) h+\sum_{E_{j} \in T_{i}} \lambda_{j}\left|u\left(E_{j}\right)-u(x)\right| \\
& \leq\left(D+\frac{c}{\nu}\right) h+\frac{L}{\nu} h .
\end{aligned}
$$

In the last inequality we used that $u$ is Lipschitz continuous with constant $L / \nu$. By (3.85) and (3.87), we complete the proof.

\section{Numerical experiments}

In this section, we present below an example to illustrate the theoretical results. The optimization problems were solved numerically by projected gradient methods, with codes developed based on AFEPack [15]. The discretization was already described in Section 2: the control function $u$ was discretized by piecewise linear but discontinuous functions, whereas the state $(y, \boldsymbol{p})$ and the co-state $(z, \boldsymbol{q})$ were approximated by the order $k=1$ Raviart-Thomas 
mixed finite element functions. In the following example, we choose the domain $\Omega=[0,1] \times[0,1], \nu=1, A=I$ and $a_{0}=0$.

Example. We consider the following two-dimensional elliptic optimal control problem

$$
\min _{u \in U_{a d}}\left\{\frac{1}{2}\left\|\boldsymbol{p}-\boldsymbol{p}_{d}\right\|^{2}+\frac{1}{2}\left\|y-y_{d}\right\|^{2}+\frac{1}{2}\|u\|^{2}\right\}
$$

subject to the state equation

$$
\operatorname{div} \boldsymbol{p}=f+u, \quad \boldsymbol{p}=-\operatorname{grad} y,
$$

where

$$
\begin{aligned}
y & =2 \sin \left(\pi x_{1}\right) \sin \left(\pi x_{2}\right), \\
z & =-\sin \left(\pi x_{1}\right) \sin \left(\pi x_{2}\right), \\
u & =\max \{a, \min (b,-z)\} \\
f & =2 \pi^{2} y-u \\
y_{d} & =y-2 \pi^{2} z \\
\boldsymbol{p} & =\boldsymbol{p}_{d}=-\left(\begin{array}{l}
2 \pi \cos \left(\pi x_{1}\right) \sin \left(\pi x_{2}\right) \\
2 \pi \sin \left(\pi x_{1}\right) \cos \left(\pi x_{2}\right)
\end{array}\right), \\
\boldsymbol{q} & =\left(\begin{array}{l}
\pi \cos \left(\pi x_{1}\right) \sin \left(\pi x_{2}\right) \\
\pi \sin \left(\pi x_{1}\right) \cos \left(\pi x_{2}\right)
\end{array}\right) .
\end{aligned}
$$

In the numerical implementation, we set $a=0.2$ and $b=0.8$ to make both the lower and the upper constraints active. In Table 1, the errors $\left\|u-u_{h}\right\|$ and $\left\|u-u_{h}\right\|_{0, \infty}$ obtained on a sequence of uniformly refined meshes are shown. Table 1 also shows the convergence orders of these errors. In Figure 1, the profile of the numerical solution of $u$ on the $64 \times 64$ mesh grid is plotted. It is easy to see that the numerical results are consistent with our theoretical analysis.

Table 1. The error of Example on a sequential uniform refined meshes.

\begin{tabular}{|c|c|c|c|c|}
\hline$h$ & $\left\|u-u_{h}\right\|$ & order & $\left\|u-u_{h}\right\|_{0, \infty}$ & order \\
\hline $1 / 16$ & $6.43573 \mathrm{e}-03$ & - & $3.39841 \mathrm{e}-02$ & - \\
\hline $1 / 32$ & $2.65667 \mathrm{e}-03$ & 1.28 & $2.07512 \mathrm{e}-02$ & 0.71 \\
\hline $1 / 64$ & $9.58166 \mathrm{e}-04$ & 1.47 & $1.14939 \mathrm{e}-02$ & 0.85 \\
\hline $1 / 128$ & $3.60974 \mathrm{e}-04$ & 1.41 & $5.89509 \mathrm{e}-03$ & 0.96 \\
\hline
\end{tabular}

\section{Conclusions}

In this paper, we discussed the order $k=1$ Raviart-Thomas mixed finite element methods for the elliptic optimal control problem (1.1)-(1.4) with pointwise control constraints. We have used piecewise linear but discontinuous functions 
instead of piecewise constant functions to approximate the control variable. We have derived $L^{2}$ - and $L^{\infty}$-error estimates for the control variable. In the future, we will investigate the $L^{\infty}$-error estimates and superconvergence of optimal control problems governed by nonlinear elliptic equations. Furthermore, we shall consider the case that $a$ and $b$ in the admissible control set $U_{a d}$ are some smooth functions (not constants as the present paper, see (1.5)).

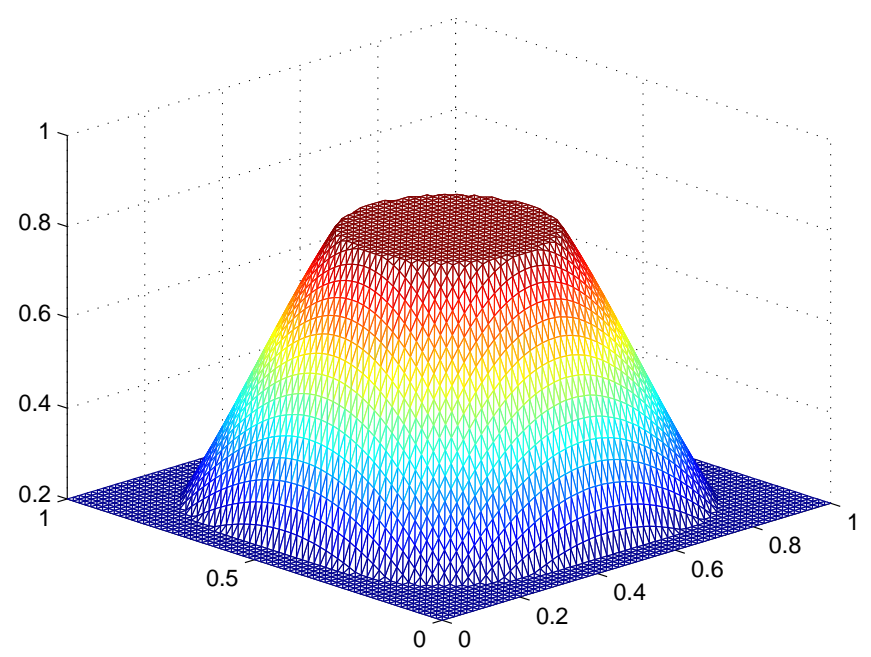

Figure 1. The profile of the numerical solution of the Example on $64 \times 64$ triangle mesh.

\section{References}

[1] N. Arada, E. Casas, and F. Tröltzsch, Error estimates for the numerical approximation of a semilinear elliptic control problem, Comput. Optim. Appl. 23 (2002), no. 2, 201229.

[2] R. Becker, H. Kapp, and R. Rannacher, Adaptive finite element methods for optimal control of partial differential equations: basic concept, SIAM J. Control Optim. 39 (2000), no. $1,113-132$

[3] F. Brezzi and M. Fortin, Mixed and Hybrid Finite Element Methods, Springer-Verlag, New York, 1991.

[4] Y. Chen, Superconvergence of mixed finite element methods for optimal control problems, Math. Comp. 77 (2008), no. 263, 1269-1291.

[5] - Superconvergence of quadratic optimal control problems by triangular mixed finite elements, Internat. J. Numer. Methods Engrg. 75 (2008), no. 8, 881-898.

[6] Y. Chen and Y. Dai, Superconvergence for optimal control problems governed by semilinear elliptic equations, J. Sci. Comput. 39 (2009), no. 2, 206-221.

[7] Y. Chen, Y. Huang, W. B. Liu, and N. Yan, Error estimates and superconvergence of mixed finite element methods for convex optimal control problems, J. Sci. Comput. 42 (2009), no. 3, 382-403. 
[8] Y. Chen and W. B. Liu, A posteriori error estimates for mixed finite element solutions of convex optimal control problems, J. Comp. Appl. Math. 211 (2008), no. 1, 76-89.

[9] P. G. Ciarlet, The Finite Element Method for Elliptic Problems, North-Holland, Amsterdam, 1978

[10] P. Grisvard, Elliptic Problems in Nonsmooth Domains, Pitman, Boston-LondonMelbourne, 1985

[11] J. Douglas and J. E. Roberts, Global estimates for mixed methods for second order elliptic equations, Math. Comp. 44 (1985), no. 169, 39-52.

[12] F. S. Falk, Approximation of a class of optimal control problems with order of convergence estimates, J. Math. Anal. Appl. 44 (1973), 28-47.

[13] T. Geveci, On the approximation of the solution of an optimal control problem governed by an elliptic equation, RAIRO. Anal. Numér. 13 (1979), no. 4, 313-328.

[14] R. Li, W. B. Liu, H. P. Ma, and T. Tang, Adaptive finite element approximation for distributed elliptic optimal control problems, SIAM J. Control Optim. 41 (2002), no. 5, 1321-1349.

[15] R. Li and W. Liu, http://circus.math.pku.edu.cn/AFEPack.

[16] J. L. Lions, Optimal Control of Systems Governed by Partial Differential Equations, Springer-Verlag, Berlin, 1971.

[17] W. B. Liu and N. N. Yan, A posteriori error analysis for convex distributed optimal control problems, Adv. Comp. Math. 15 (2001), 285-309.

[18] Z. Lu and Y. Chen, $L^{\infty}$-error estimates of triangular mixed finite element methods for optimal control problems governed by semilinear elliptic equations, Numer. Anal. Appl. 2 (2009), no. 1, 74-86.

[19] C. Meyer and A. Rösch, Superconvergence properties of optimal control problems, SIAM J. Control Optim. 43 (2004), no. 3, 970-985.

[20] $L^{\infty}$-error estimates for approximated optimal control problems, SIAM J. Control Optim. 44 (2005), 1636-1649.

[21] P. A. Raviart and J. M. Thomas, A mixed finite element method for 2nd order elliptic problems, aspects of the finite element method, Lecture Notes in Math, Springer, Berlin, 606 (1977), 292-315.

[22] X. Xing and Y. Chen, $L^{\infty}$-error estimates for general optimal control problem by mixed finite element methods, Int. J. Numer. Anal. Model. 5 (2008), no. 3, 441-456.

Key Laboratory for Nonlinear Science and System Structure

School of Mathematics and Statistics

Chongqing Three Gorges University

Wanzhou 404100, Chongqing, P. R. China

E-mail address: htlchb@163.com 